\title{
Experiencias adaptativas de líderes de influencia a entornos virtuales: una perspectiva de aprendizaje en pandemia
}

\section{Adaptative experiences of influential leaders in virtual environments: a pandemic learning perspective}

Experiências adaptativas de líderes influentes em ambientes virtuais: uma perspectiva de aprendizagem

\section{Delia Fernández Roncal \\ deliafernandez@upeu.edu.pe \\ https://orcid.org/0000-0002-3214-8187}

Escuela de Posgrado, Universidad Peruana Unión Lima, Perú

Yván Martín Balabarca Cárdenas

yvanbalabarca@upeu.edu.pe

https://orcid.org/0000-0002-2955-2816

Facultad de Teología, Universidad Peruana Unión Lima, Perú

\section{Victoria Martinez Tejada}

vickyjs@upeu.edu.pe

https://orcid.org/0000-0001-8474-4784

Escuela de Posgrado, Universidad Peruana Unión Lima, Perú

Josué Turpo Chaparro

josuetc@upeu.edu.pe

https://orcid.org/0000-0002-1066-6389

Escuela de Posgrado, Universidad Peruana Unión Lima, Perú

\section{Segundo Salatiel Malca Peralta \\ segundomalca@upeu.edu.pe \\ https://orcid.org/0000-0001-6294-1541}

Facultad de Ciencias Humanas y Educación, Universidad Peruana Unión Lima, Perú

\section{RESUMEN}

El objetivo del estudio fue validar experiencias adaptativas en tres aspectos: procesos de enseñanza-aprendizaje, manejo de herramientas digitales y el impacto en el aspecto personal. El estudio es de enfoque cualitativo, con un proceso de investigación-acción participativa durante la pandemia; cómo la adaptación de forma deliberada e involuntaria generó exigencia al afrontamiento de la incertidumbre, la aceptación de reinventarse en el ejercicio de la influencia en una nueva situación, llevó a tomar los recursos ofrecidos, partiendo desde lo conocido hacia lo desconocido y en ocasiones desde lo desconocido para mantener lo conocido, dejando ver una línea de pensamiento, construida desde impresiones, acciones inductivas y deductivas. La información fue recogida a través de entrevistas semiestructuradas a 15 líderes de influencia, vía plataforma Zoom. Los resultados mostraron que las experiencias adaptativas dejaron un potencial digital para el proceso de enseñanza-aprendizaje, con un particular impacto personal.

Palabras clave: Aprendizaje; Enseñanza; Entornos virtuales; Experiencias adaptativas

\section{ABSTRACT}

The objective of the study was to validate adaptive experiences in three aspects: teaching-learning processes, handling of digital tools and the impact on the personal aspect. The study has a qualitative approach, with a participatory action research process during the pandemic; how the deliberate and involuntary adaptation generated demand to face uncertainty, the acceptance of reinventing oneself in the exercise of influence in a new situation, led to taking the resources offered, starting from the known to the unknown and sometimes from the unknown to maintain the known, revealing a line of thought, built from impressions, inductive and deductive actions. The information was collected through semistructured interviews with 15 influential leaders, via the Zoom platform. The results showed that the adaptive experiences left a digital potential for the teaching-learning process, with a particular personal impact.

Key words: Learning; Teaching; Virtual environments, Adaptive experiences

\section{RESUMO}

O objetivo do estudo foi validar experiências adaptativas em três aspectos: processos de ensino-aprendizagem, manuseio de ferramentas digitais e o impacto no aspecto pessoal. O estudo tem abordagem qualitativa, com processo de pesquisa-ação participativa durante a pandemia; como a adaptação deliberada e involuntária gerou demanda para enfrentar a incerteza, a aceitação de se reinventar no exercício de influência em uma nova situação, levou a tomar os recursos oferecidos, partindo do conhecido para o desconhecido e às vezes do desconhecido para manter o conhecido, revelando uma linha de pensamento, construída a partir de impressões, ações indutivas e dedutivas. As informações foram coletadas por meio de entrevistas semiestruturadas com 15 líderes influentes, por meio da plataforma Zoom. Os resultados mostraram que as experiências adaptativas deixaram um potencial digital para o processo ensino-aprendizagem, com particular impacto pessoal.

Palavras-chave: Aprendizagem; Ensino; Ambientes virtuais; Experiências adaptativas 


\section{INTRODUCCIÓN}

Luego de validar las experiencias adaptativas alcanzadas durante la pandemia, el análisis permitió ver las alternativas tomadas y creadas por los participantes en la construcción de los entornos virtuales, los cuales facilitaron la enseñanza y la comunicación (Cuervo, 2021) fusionando la incipiente experiencia digital con las iniciativas de influencia en el liderazgo para el alcance de las metas. Asimismo, la carencia de recursos pedagógicos estructurados para el mundo digital, considerando que naturalmente las tradicionales formas presenciales de comunicación fueron cercenadas por la Covid-19 (Leal, 2021) dieron pie a novedosas experiencias participativas de adaptación creativa como acciones emergentes, a consecuencia del cierre masivo de las actividades presenciales a nivel mundial (Valverde, 2021).

Por lo tanto, a fin de mitigar la propagación y el impacto del virus, los facilitadores hicieron uso de las redes sociales para seguir con las horas lectivas, aumentando las asesorías a los liderados y ahorrando en tiempo de desplazamientos (Cuervo, 2021). A pesar de las limitaciones la comunicación verbal y no verbal reemplazaron el contacto personal en el proceso educativo y las redes desempeñaron un rol fundamental (Giraldo, Gómez y Giraldo, 2021). Por ende, los facilitadores de la enseñanza desplegaron diversas modalidades de aprendizaje a distancia, mediante una diversidad de formatos y plataformas para impactar en las mentes de niños, jóvenes y adultos, manteniendo los estándares de la realidad presencial (Cerbino y Ángulo, 2020).

No obstante, la poca experiencia en entornos virtuales entre otras limitaciones, forzaron a los facilitadores a innovar en sus estrategias y metodologías de enseñanza-aprendizaje (García, 2020), a pesar de la urgencia y el poco tiempo para adaptarse a estos nuevos entornos (Morales et al., 2020). Este forzoso cambio de lo presencial a lo virtual, dejó una enriquecedora experiencia y una nueva perspectiva sobre la virtualidad; no solo desde el escenario mismo que permite aprender y enseñar, sino también desde los cambios emocionales, las limitaciones de no socialización y el estado de alarma y de alerta, dejando notables consideraciones a tener en cuenta.

Ahora bien, la modalidad de educación a distancia significó durante la pandemia del Covid-19 una migración fundamentalmente hacia el soporte digital (Leal, 2021). Estos nuevos entornos virtuales ofrecieron soluciones de emergencia para afrontar las crisis que se generaron por el confinamiento; estrategias a la que recurrieron muchos gobiernos para frenar el contagio de este nuevo virus (García, 2020). Por lo tanto, corresponde reflexionar sobre las percepciones que se generaron durante esta experiencia en las líderes de influencia.

Por un lado, el liderazgo al frente de escenarios de aprendizaje se vio afectado y dejó ver escasas habilidades y destrezas de parte de las líderes de influencia. Desde las técnicas asumidas y el impacto personal (RPP, 2021), hasta la optimización progresiva, como sucedió en Perú, donde a pesar de la enorme brecha digital, el $32.1 \%$ de los hogares contó con una computadora (El Comercio, 2020). En efecto, asumir y reinventarse a los entornos virtuales, dio paso a la adaptación en los comportamientos para afrontar diferentes brechas (Cerbino y Ángulo, 2020), ya que una entrevista realizada a 3000 profesores sobre sus experiencias de enseñanza-aprendizaje durante la pandemia del COVID-19, el 71\% pensó que el celular y el WhatsApp no eran ideales para la comunicación entre padres y docentes; además, la mayoría creía que la educación efectuada de este modo acentuaba desigualdades (Näslund et al., 2020). 
Por lo tanto, la importancia de enseñar y aprender en entornos oportunos y fluidos de comunicación, dejó ver la necesidad de conocer otras formas de adaptación en una mejora continua, para sumar experiencias en quienes forman a los estudiantes. Esto se constituyó en un desafío, dado que la principal dificultad fue la comunicación con los participantes y sus familias (Aragón y Cruz, 2020). Por otro lado, se hizo notoria en las líderes de influencia la realidad de asumir la conducción del hogar en paralelo con la enseñanza sin líneas divisorias. El trabajo remoto borró las fronteras entre los dos ámbitos, ya que el trabajo se realizó desde casa y con la presencia de niños (Furman, 2020). Este ejercicio demandó mayor adaptación, tanto en quienes recibían el aprendizaje como en los que lo impartían (Pedraza, 2021).

El presente estudio centró el interés en el registro de la adaptación y las acciones que hicieron posible mantener el conocido control y la interacción de los procesos, tanto de inicio, de sostenibilidad y de motivación constante para los liderados y las líderes de influencia. Como resultado del diagnóstico de necesidades, se observó que el desarrollo de la pandemia produjo un impacto personal, mental y físico en las personas. Esta situación generó la necesidad de observar la adaptación de las líderes de influencia en escenarios de enseñanza-aprendizaje (Dos Santos, Scorsolini-Comin y Marchi, 2020).

Se observó también que la inesperada coyuntura entorpece el proceso regular de influencia personal y expone la capacidad para afrontar la incertidumbre de las líderes de influencia, haciendo cambios que conllevan a la adaptación, de lo contrario serían meros observadores pasivos del estancamiento del proceso de enseñanza-aprendizaje. Tal situación provocó emociones fuertes y agotamiento mental en quienes ejercían influencia, afectando el trabajo y el bienestar de las personas cercanas y de la comunidad (Celio, 2021). Por lo que resulta necesario desarrollar mayor resiliencia y manejo del estrés (Santamaría, 2020).

Asimismo, repercutió en el forzado uso de las herramientas digitales, siendolas más usadas Google, Meet, BlueJeans y Zoom (Cerbino y Ángulo, 2020). Estas herramientas constituyeron un medio para salvaguardar el proceso cotidiano en distintas áreas del quehacer humano, y en especial en la educación (Castro e Hidalgo, 2021). En el contexto peruano, las redes sociales más utilizadas fueron Facebook con $72 \%$, Instagram $21.5 \%$, y Twitter y YouTube con 3\% y 3.5\% respectivamente (Andina, 2021). No obstante, a pesar de las facilidades ofrecidas por estas plataformas, los facilitadores a nivel global, reportaron ciertas dificultades de conexión con los liderados por la falta de respuesta no verbal, la poca motivación y la carencia de espacios de estudio (Castro e Hidalgo, 2021). Desafíos constantes que debían superar las líderes de influencia durante la pandemia.

\section{MÉTODO}

Se realizó un estudio cualitativo con un proceso de análisis de investigación-acción participativa, con la intención de comprender las experiencias adaptativas en tres aspectos: procesos de enseñanza 
y aprendizaje, manejo de herramientas digitales y el impacto en el aspecto personal. Se trabajó bajo un enfoque cualitativo, con un proceso de análisis de investigación acción participativa, el cual se construyó luego del acopio de datos narrativos en el contexto de la pandemia. El foco reside en los valores del profesional investigador y está basado en las personas, ya que se investigan sus acciones y los investigados se convierten en coinvestigadores (Colmenares, 2017) y el investigador es el instrumento mismo.

El tipo de participación fue contractual, dado que existió un común acuerdo para el uso de datos, donde el investigador es considerado como el “dueño" de los resultados y los participantes actúan como informalmente "contratados" (Zapata y Vidal, 2016). El interés de estudio fue validar la adaptación de las líderes de influencia en entornos virtuales, para lo cual se desarrolló una exhaustiva revisión de artículos de rigor académico, tanto en Scopus como en las distintas revistas indexadas (Scielo, Redalyc, Dialnet, entre otras). Se seleccionaron artículos que presentaban aproximación a las unidades de análisis de interés entre el 2020-2021, considerando el contexto en el que se desarrolló el estudio. Además, se elaboró una entrevista semiestructurada, cuyo análisis fue a través de una triangulación y se redactó las preguntas reactivas para averiguar las acciones de adaptación que experimentaron las informantes.

El proceso de análisis se hizo a través de anotaciones de observación directa, anotaciones interpretativas y anotaciones personales, las cuales se desarrollaron a través de los investigadores y se sistematizó mediante una tabla de doble entrada en relación con las unidades de análisis y anotaciones finales, que fueron alcanzadas mediante la triangulación entre los investigadores por separado, la entrevistadora investigadora y los testigos.

Como se aprecia en la Figura 1, las experiencias adaptativas fueron el centro del presente estudio en las cuales las anotaciones se dieron en dos sentidos, mediante realidades y acciones que ingresaron a formar parte de tales experiencias y regresaron en experiencias cotidianas de las líderes, mediante la adaptación a diversas situaciones, el manejo de herramientas digitales en la enseñanza-aprendizaje y en el impacto personal. 


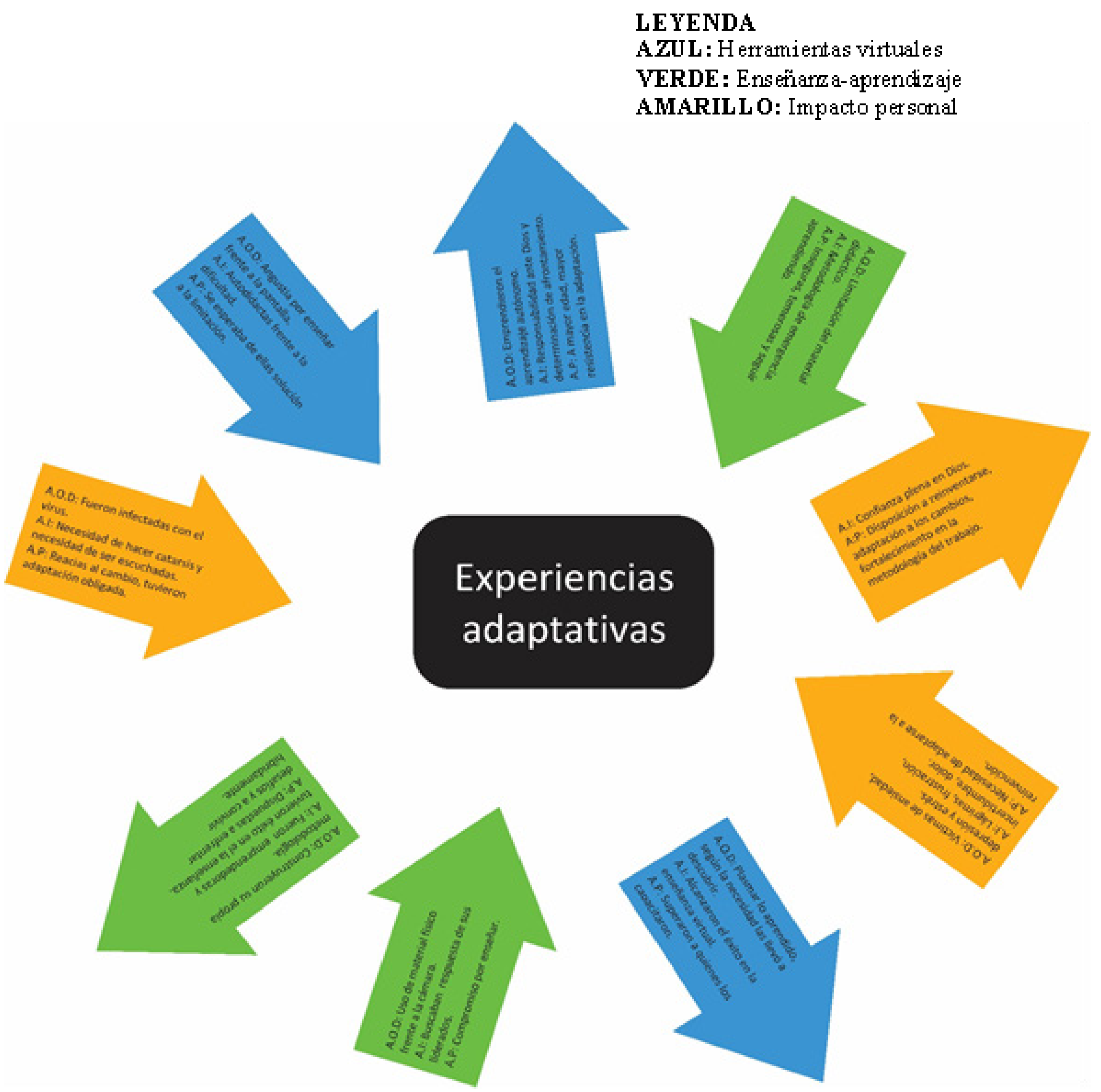

A.O.D: Anotaciones de observación directa A.I: Anotaciones interpretativas

A.P: Anotaciones personales

Figura 1. Diagrama de anotaciones de entrada y salida de las experiencias adaptativas. 
Con la información alcanzada se generó la relación de asociación entre los principales eventos que se dieron durante la pandemia a nivel nacional y sus repercusiones en la población, dejando ver el impacto en el comportamiento de las líderes de influencia y sus acciones de adaptación. Estos sucesos quedaron gráficamente plasmados en un organizador gráfico de información compartido en los resultados.

El estudo se realizó durante los meses de abril a noviembre de 2021, en el que participaron 15 líderes de Lima Este-Perú, los cuales ejercieron su liderazgo sobre 14,560 personas. Un $80 \%$ fueron casados y tuvieron una edad promedio de 43 años.

Las participantes se dedicaron a ejercer su liderazgo en la enseñanza y el aprendizaje por pedido de su organización de base. El 47\% sufrió de una afección relacionada al COVID-19 durante ese año y el 93\% sufrió por la muerte de uno o más familiares, especialmente en los meses de junio a agosto en donde la pandemia alcanzó su pico máximo.

El criterio de selección fue por: metas alcanzadas, pertenencia a las diferentes franjas etáreas, visibilidad en redes sociales, obtención de una mayor cantidad de réplicas de cada campaña o actividad en sus lugares de influencia, contar con excelentes referencias de sus líderes respecto al desempeño en su trabajo, ser consideradas el brazo derecho de su líder superior inmediato, capacidad de movilización de sus liderados, mostrar tener iniciativa, y orientación al emprendimiento.
Se hizo la observación a través de una entrevista semiestructurada, la misma quefuediseñaday creada Ad hoc por los investigadores y fue aplicada a cada líder de influencia, a través de videoconferencias por la plataforma Zoom. La muestra estuvo constituida por 1 líder macroregional, 2 líderes regionales, 6 líderes distritales y 6 líderes locales de la Asociación Peruana Central Este, su organización de base.

En primera instancia se invitó a los participantes a través de llamadas telefónicas y WhatsApp, las 15 personas dieron su consentimiento informado a través de un formulario electrónico (Google form), para grabar las entrevistas en presencia de un testigo y para posteriormente analizar la información. Respondieron 10 preguntas que podían desencadenar repreguntas, en un tiempo estimado de 50 minutos a 1 hora; la imagen se tomó con un golpe de cámara de plano medio que permitió observar todo gesto, desde las manos hasta el rostro y contornos. Para el desarrollo de la entrevista las líderes se ubicaron en el mismo lugar donde hacían las transmisiones a sus liderados en los procesos de enseñanza-aprendizaje; cada entrevista tuvo la presencia anónima de una tercera persona como testigo, quien estuvo todo el tiempo con cámara apagada, y tomó notas de sus apreciaciones en una guía de observación. Al iniciar la investigadora se presentó y presentó el título y objetivo de la investigación de la cual formarían parte cada una de las informantes y posteriormente fueron analizadas asíncronamente. 
En esta investigación la entrevista arribó a respuestas que fueron analizadas en una tabla diseñada por los investigadores, en la cual se tabularon por frecuencia para concluir en los impactos recibidos por las líderes de influencia; además de triangular la información pues se acompañó con una ficha de sistematización para el análisis, tanto de parte de los investigadores como de los testigos, para mantener la confiabilidad de la información, generando finalmente un análisis completo. Finalmente, se generó un organizador gráfico de información llamado línea de tiempo, donde se visualizaron los eventos que desencadenaron mayor demanda de adaptación en las líderes entrevistadas y sus reacciones, logrando generar y explicar posibles acciones en tal relación de asociación de experiencias, todas ellas en fechas del proceso pandémico en el país, y los comportamientos en pandemia, el resultado es de gran valor para futuras investigaciones y para la toma de decisiones de quienes ocupan puestos determinantes al fomentar con estos hallazgos un liderazgo y educación sostenible.

\section{RESULTADOS}

El análisis de datos siguió el modelo propuesto por Creswell y Creswell (2017), mediante un diseño concurrente, donde básicamente se suman análisis realizados en paralelo. Los hallazgos conllevan a declarar que durante el manejo emocional de la pandemia, el $60 \%$ de los participantes, se centró en dos emociones distinguidas: el miedo y la preocupación; el primero, por enfrentar la enseñanza-aprendizaje, la realidad del manejo de herramientas virtuales y el impacto personal en un escenario amenazante en su liderazgo por la posible pérdida de la salud y cómo mantenerla en condiciones de confinamiento; la segunda preocupación fue la emoción, producto del querer adelantarse a nuevas experiencias, sin saber cómo se comportan las mismas.

Estos hallazgos se encontraron luego de que se codificaron las entrevistas como Experiencia Adaptativa 01 (EA01), hasta la EA15. Luego las respuestas se agruparon en los aspectos: impacto en la enseñanza aprendizaje; impacto en el uso de las herramientas virtuales, e impacto personal. Cada una de las agrupaciones generaron tendencias que se graficaron en las figuras que se exponen en el artículo. Los impactos en estas tres áreas se midieron a través de las preguntas de la entrevista.

Las entrevistas demostraron que la pandemia provocó emociones fuertes y agotamiento mental, afectando el trabajo y bienestar de las líderes de influencia, y el bienestar de las personas cercanas a ellos y de la comunidad. Por lo que fue trascendental el desarrollo de resiliencia y manejo del estrés (CDC, 2020). Esto se vio reflejado en las actividades de las líderes de influencia, ya que la presencia de tales emociones desencadenó problemas de salud física como dolores en la columna vertebral, gastritis, hígado graso, y en un caso muy particular mioma en el seno.

A nivel psicológico, reportaron haber sentido temor como la emoción más mencionada. Lo que máslas impactó fue el no poder asistir a sus reuniones 
presenciales tanto para recibir enseñanzas o para compartir aprendizajes. Otro aspecto psicológico importante fue la adaptación al encierro, con sus respectivos recursos personales de aceptación y soporte; los meses en que más se sintieron afectadas fue entre marzo a mayo, donde acusaron síntomas de las enfermedades referidas.

En cuanto a las aplicaciones que las líderes usaron para poder mantener el ejercicio de su influencia se encentran WhatsApp, Google Meet, Facebook, Zoom y YouTube, dejando de lado el uso de Instagram, Snapchat, Telegram, Discord y otros de uso cotidiano en la enseñanza-aprendizaje, pues el $73 \%$ es ama de casa, a pesar de que el 93\% mencionó tener estudios superiores.

Por otro lado, la plataforma Zoom fue la que más se utilizó como medio de videoconferencia, debido al uso corporativo por parte de su organización; sin embargo, Google Meet por ser gratuita, fue la que llegó a tener mayor relevancia para esfuerzos individuales al comienzo de la pandemia.

La adaptación en el manejo de herramientas virtuales, el nuevo escenario de enseñanzaaprendizaje y el cómo sobrellevar el impacto personal, tomó un tiempo estimado de 3 a 6 meses, entre los meses de junio y noviembre, al cabo del cual el $60 \%$ de las líderes de influencia llegaron a manejar muy bien las tecnologías de comunicación y el 40\% aún quedó en crecimiento, dejando en evidencia que la adaptación para la enseñanza en entornos virtuales es un proceso que toma su tiempo.
Durante las entrevistas muchas líderes de influencia aceptaron que tuvieron que dedicarse a capacitar a sus liderados antes de influenciar en ellos, a fin de que puedan acceder a los medios de comunicación digital, haciendo mayor el desafío pues pasaban a ser entrenadoras instrumentales antes de otorgar la enseñanza, para asegurar el aprendizaje, ya que varias de las líderes como muchos de sus liderados eran mayores en edad; por lo tanto, no tenían el interés ni las destrezas para utilizar las plataformas digitales. Por otro lado, algunas líderes jóvenes no tuvieron los recursos financieros para pagar una línea de internet, ya que tenían dependencia económica y necesitaban maximizar los datos de sus celulares.

\section{Discusión}

Por lo señalado en este estudio, se puede afirmar que la pandemia por COVID-19 generó una crisis global y aún más acentuada en Perú, el cual tuvo la mayor tasa de mortalidad por Covid-19 en el mundo, con 6065 muertos por cada millón de habitantes (France 24, 2021). Con el cierre masivo de las actividades presenciales en más de 190 países, a fin de mitigar la propagación y el impacto del virus (CEPAL, 2020), la UNESCO detectó grandes brechas en cuanto a la distribución de los facilitadores en general y de los facilitadores mejor calificados, en especial en países y regiones con menores ingresos, más aún en las regiones rurales (Pedraza, 2021). En consecuencia, las líderes de influencia desplegaron diversas modalidades de 
enseñanza a distancia, mediante la utilización de una diversidad de aplicaciones digitales y plataformas virtuales (Conexiónesan, 2020).

Como estrategia adaptativa usaron técnicas tradicionales, de realidad presencial frente a una cámara de videoconferencia, siendo el entorno digital un vehículo para seguir con sus estrategias familiares, lo cual ocasionó que no hicieran uso total de las tecnologías, sino solo como proyectores del uso clásico de las actividades instrumentales comunes, a consecuencia de la migración de la enseñanza y aprendizaje presencial a la realidad online. Lo cual creó un cambio en los paradigmas de quienes ejercen enseñanza. Este proceso mostró que la realidad tradicional en la enseñanza se adaptó durante la pandemia, marcando un antes y un después, ya que para las líderes de influencia fue más sencillo partir de lo conocido hacia lo desconocido, en el dominio de lo que se usaba y funcionaba en la presencialidad, lo cual fue relevante para la adaptación en el uso de las herramientas virtuales, conocida como adaptación inductiva.

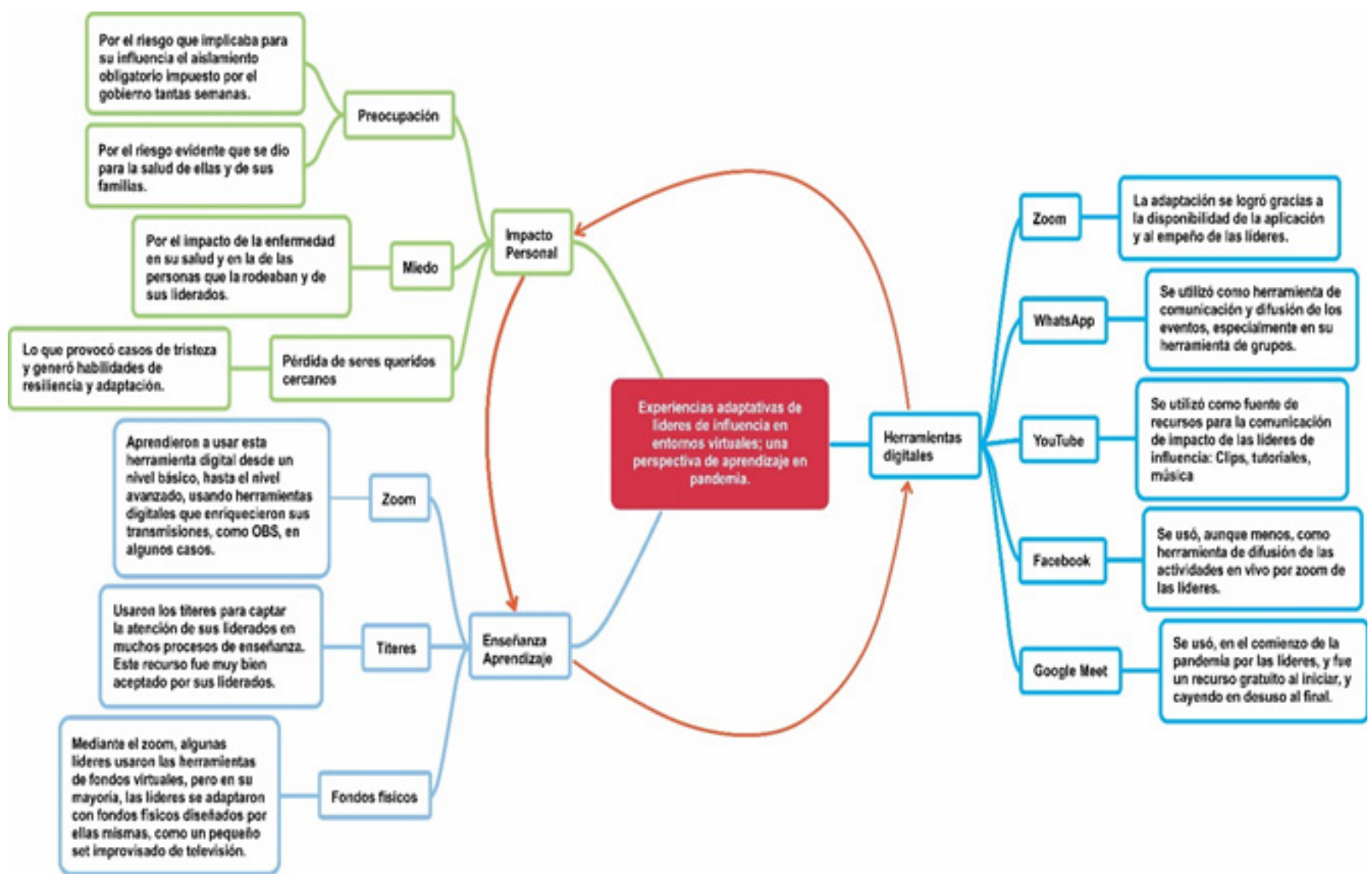

Figura 2. Experiencias adaptativas de líderes de influencia en entornos virtuales: una perspectiva de aprendizaje en pandemia. 
En esta crisis las líderes de influencia mostraron liderazgo e innovación para asegurar el aprendizaje, garantizando que el proceso continúe y ningún influenciado se quede relegado, considerando que la pandemia afectó a más del $90 \%$ de la población estudiantil mundial (1600 millones de estudiantes). Esta coyuntura ha agravado las desigualdades por las condiciones de acceso a dispositivos móviles y a una red de internet estable con buen ancho de banda, la cual está directamente relacionada con la capacidad de pago (ONU, 2020). Esta realidad es semejante a la de México, donde solo el $44.3 \%$ de los hogares contó con una computadora, el 56,4\% tuvo conexión a internet, y el 10,7\% accedió a una red fuera de su hogar (INEGI, 2019). Mientras que, en Perú, solo el $40.1 \%$ de los hogares tuvo acceso a internet (El Comercio, 2020).

En líneas generales las desventajas del contexto peruano, añadió mayor dificultad al pasar a los entornos virtuales con tecnologías nuevas, porque ello demandó costumbres, relaciones, y conocimientos para articular las herramientas a procesos históricos personales y colectivos, conformando entornos y ámbitos de aprendizaje (Lovón y Cisneros, 2020). Todos estos procesos se aceleraron en las líderes de influencia, que, al finalizar el año en pandemia, partiendo de las experiencias previas para los nuevos desafíos de su labor, integraron el nuevo conocimiento a sus prácticas habituales.

Asimismo, cuando se trata de enseñar a distancia, se necesita redefinir el para qué de la educación, observando que el aprendizaje y las TIC generan múltiples lenguajes, debido a las trayectorias individuales de aprendizaje, la ubicuidad del mismo y la personalización del entorno digital (Torres,
2017). Desde las características del lugar desde donde se hacen las transmisiones, encontrar un fondo adecuado, aprender a gestionar los recursos para elegir los backgrounds digitales a usar, aprender a armar sus propios backgrounds (que son los fondos observados al proyectarse frente a la cámara con elementos de su interés en la enseñanza), la luz necesaria, el lenguaje de los emoticones como nuevos términos de comunicación, la conexión de internet que hizo lenta su transmisión y la de su audiencia que se tradujo en pantallas congeladas, voces entrecortadas (que hacían perder la emociones en la comunicación), los mensajes por chats que llegaban a destiempo en una intención de comunicación, el hablar a una pantalla en lugar de una conexión tradicional en la enseñanza, y la entrada y salida de los usuarios a las salas de videoconferencia durante los tiempos del enseñar y el aprender.

Esta personalización se hizo patente desde la perspectiva de las experiencias previas, como lo declaró Coll; cada líder de influencia optó por adaptarse de la manera que sus experiencias previas les permitieron. Esto se observó en las entrevistas que se optó por seguir con la enseñanza clásica, pero de manera televisada, por lo menos en los primeros meses. A partir de la mitad de la experiencia en pandemia, se logró alcanzar un nivel intermedio de pericia, logrando desarrollar un embedded Learning/embedded teaching alcanzando a desarrollar la esperada influencia.

Por otro lado, Perú reportó 200000 muertes hasta el mes de octubre del año 2020 durante la pandemia de COVID-19, alcanzando un récord de 6,058 muertes por cada millón de personas (Grillo y Romero, 2020), esto explica el miedo y la preocupación de las líderes de influencia, ya que 
los reportes de muertes y contagios al inicio de la pandemia no coincidían con la experiencia que se estaba viviendo.

Según una entrevista a 3000 profesores, para que compartan sus experiencias de aprendizaje remoto durante la pandemia del COVID-19, se encontró que el $71 \%$ pensaba que el teléfono móvil y el WhatsApp no eran las plataformas ideales para la comunicación entre padres y docentes; además les preocupaba que la educación a distancia estaba acentuando las desigualdades. Esto se hizo evidente cuando las líderes de influencia entrevistadas en el estudio notificaron que fueron muchos los hogares que no se podían conectar a los esfuerzos de comunicación que se ejecutaban.

En Perú se evidenció que la comunicación de los líderes en el contexto educativo tuvo dificultades entre los estudiantes y su entorno familiar (Aragón y Cruz, 2020), puesto que la brecha tecnológica y generacional dejó su impacto en la permanencia y sostenibilidad de la comunicación, tanto en los liderados como en los propios líderes en el proceso de adaptación. Asimismo, además de ejercer el teletrabajo, los líderes debieron atender obligaciones domésticas, lo cual demandó mayor inversión de tiempo e imposibilidad para cumplir las metas, generando una intermitencia entre los quehaceres del trabajo y del hogar (BBC, 2020).

Por otro lado, un proceso implícito fue el sortear acciones de adaptación en horarios, jornadas, tiempos y pericia, pues desarrollar el quehacer doméstico unido al desempeño en el escenario virtual demandó ajustes de tales acciones desde ambas realidades, obligando a optimizar el tiempo. Las líderes aprendieron a convivir unidas en una misma experiencia personal.
Las herramientas de videollamadas más usadas durante la pandemia fueron Zoom, Google Meet, Skype, entre otras, estas crecieron en 53\% respecto al año 2019; mientras que para la gestión se utilizó en mayor proporción WhatsApp y Gmail (Gómez, 2021). Esto coincide con los recursos que usaron las líderes de influencia, añadiendo el YouTube a las herramientas antes mencionados.

Respecto a las redes sociales más usadas durante la pandemia, YouTube y Facebook fueron las de mayor preferencia por los adultos mayores de 25 años de edad, y entre los jóvenes de 18 y 24 años Instagram (Gestión, 2020). Mientras que las líderes de influencia que tuvieron una edad promedio de 43 años, utilizaron Facebook como herramienta principal de comunicación.

La educación en pandemia y todos los elementos que aparecen en esta realidad, en sus diferentes escenarios, permitieron afirmar que las adaptaciones en la enseñanza y el aprendizaje, más allá de hacer uso oportuno, sistemático y creativo de las herramientas digitales, deja un claro escenario de cultura transformada en la sociedad peruana, donde el aprender, desaprender y aprender forman parte de innegables experiencias adaptativas, para seguir influenciando no solo con buenas sino también con esperadas prácticas.

\section{CONCLUSIONES}

La gestión del conocimiento es muy importante en los entornos educativos, y con la pandemia se descubrieron los siguientes aspectos:

\section{Enseñanza y aprendizaje}

A fin de poder ejercer la influencia en el proceso de enseñanza y aprendizaje, las entrevistadas evidenciaron que fueron capaces de pasar de la 
presencialidad a una nueva manera de ejercerla, con adaptaciones serias y desafiantes, en forma, en sostenibilidad y en entrenamientos, antes de brindar la enseñanza y recibir nuevos aprendizajes para ejercer su influencia. Sintieron alegría de haber podido aprovechar el tiempo para aprender nuevas maneras de enseñar y aprender, llegando a sentirse desafiadas para poder aprender más sobre nuevas tecnologías y métodos de enseñanza aprendizaje para educar a sus liderados.

Asimismo, la plataforma Zoom fue el mejor vehículo para tal proceso pedagógico, pues desde su experiencia permitió utilizar recursos que ellas dominaban, como títeres, fondos de titiritero, porque solo 2 de las 15 líderes hicieron uso constante de los fondos virtuales (backgrounds) al comienzo de la pandemia: pero, al final de la misma 14 usaron los fondos virtuales además de sus herramientas físicas.

Las líderes reportaron que utilizaron medios físicos en respuesta al reporte de los liderados, quienes expresaron que gustaban de interacciones comunicativas con medios físicos que ellos pudieran percibir a través de las cámaras y posteriormente reproducir en sus hogares durante el aislamiento que la pandemia provocó, tales reproducciones no eran precisamente digitales.

\section{Herramientas digitales}

A pesar de que las líderes de influencia reportaron preocupación al comienzo de la pandemia, con el transcurrir del tiempo aprendieron a usar los diferentes medios virtuales, percibiéndolos como oportunidades y acciones innovadoras. En este proceso fue importante el apoyo de familiares cercanos como hijos o cónyuges, con quienes compartían el mandato de cuarentena y aislamiento social, así como algunos jóvenes (practicantes universitarios) quienes les ayudaron a producir las sesiones de enseñanza y a compartir transmisiones por diferentes medios de comunicación, especialmente los primeros 3 a 6 meses.

Los medios virtuales más usados por las líderes de influencia fueron: WhatsApp, Zoom, YouTube, Facebook, Power Point y Google Meet. Mientras que los menos utilizados: Twitter, Kahoot, OBS y Canva; motivo por el cual se concluye que el innovar en herramientas digitales fue de gran utilidad para que el proceso de enseñanza-aprendizaje sea un proceso amistoso y efectivo.

\section{Impacto personal}

En cuanto al impacto personal a nivel biológico, 8 entrevistados reportaron que no se enfermaron, 7 sufrieron alguna enfermedad reportada en la entrevista y 14 participantes reportaron que se lograron adaptar a la pandemia con y sin enfermedad. Lo que las animó contundentemente en tal situación fue pensar en el cuidado de Dios, su responsabilidad, y su familia. Estos pensamientos les permitieron continuar con sus labores y metas y superar las dificultades.

Asimismo, en las analizadas experiencias de adaptación, las acciones encontradas dejaron en evidencia que la adversidad se transformó en un motor, acción esperada en el perfil de un enseñante, que se alimenta de cada dificultad y que ve en ellas un potencial de crecimiento. Por lo tanto, es necesario exponer a los que lideran los procesos de enseñanza-aprendizaje a experiencias adaptativas que mantengan viva la ventaja humana del aprendizaje. 


\section{REFERENCIAS}

Andina. (2021). Facebook e Instagram son las redes sociales donde más interactúan los peruanos. Retrieved December 8, 2021, from https://andina.pe/agencia/noticia-facebook-einstagram-son-las-redes-sociales-donde-masinteractuan-los-peruanos-839307.aspx

Aragón, J., y Cruz, M. (2020). El año de las maestras y maestros en Perú. Retrieved December 8, 2021, from https://escuela.pucp.edu.pe/gobierno/ investigacion/reportes-tematicos-2/2020-elano-de-las-maestras-y-maestros-en-el-peru/

BBC. (2020). Clases en Zoom: 4 problemas de la enseñanza en línea que señala el profesor que anunció su renuncia a sus alumnos en directo. Retrieved December 8, 2021, from https://www. bbc.com/mundo/noticias-54787845

Castro, N.; y Hidalgo, P. (2021). Comunicación y nuevas realidades: aprendizajes en contextos de pandemia. Revista Enfoques de La Comunicación, (5). Retrieved from https:// repositorio.consejodecomunicacion.gob.ec/ bitstream/CONSEJO_REP/110/1/Educación Transmedia - Confinamiento\%2C Aprendizaje y Nuevas Plataformas.pdf

CDC, C. para el control y la prevención de enfermedades. (2020). Empleados: cómo manejar el estrés laboral y aumentar la resiliencia durante la pandemia del COVID-19. Enfermedad Del Coronavirus 2019. Retrieved from https://espanol.cdc.gov/coronavirus/2019ncov/community/mental-health-nonhealthcare.html

Celio Pillaca, J. (2021). Burnout y satisfacción con la vida en docentes que realizan clases virtuales en un contexto de pandemia por covid-19. Puriq, 3(1), 185-212. https://doi.org/10.37073/ puriq.3.1.142

Cerbino, M., y Ángulo, N. (2020). La construcción social de plataformas digitales y la experiencia de la vida cotidiana ¿cómo funcionan los objetos técnicos en época de confinamiento social? Chasqui Revista Latinoamericana de Comunicación, 1(143), 295-318. https://doi. org/10.16921/chasqui.v0i143.4305
Colmenares Escalona, A. M. (2017). Enalteciendo las competencias investigativas de los docentes de la Universidad Pedagógica Experimental Libertador, Instituto Pedagógico de Barquisimeto. Voces y Silencios. Revista Latinoamericana de Educación, 8(2), 35-52. https://doi.org/10.18175/vys8.2.2017.03

Conexiónesan. (2020). Educación online: retos que enfrentan los docentes para adaptarse a la "nueva normalidad." Retrieved December 9, 2021, from https://www.esan.edu.pe/apuntesempresariales/2020/06/educacion-online-retosque-enfrentan-los-docentes-para-adaptarse-ala-nueva-normalidad/

Creswell, JW y Creswell, JD (2017). Diseño de investigación: enfoques cualitativos, cuantitativos $y$ de métodos mixtos. Publicaciones sabias.

Cuervo Méndez, I. M. (2021). Retos de la práctica pedagógica en tiempos de confinamiento mediada por entornos digitales. Revista Estudios, 43(43). Retrieved from https:// revistas.ucr.ac.cr/index.php/estudios/article/ download/49349/49176

Dos Santos Santiago Ribeiro, B. M., ScorsoliniComin, F., y De Marchi Barcellos Dalri, R. de C. (2020). Ser docente en el contexto de la pandemia de COVID-19: reflexiones sobre la salud mental. Index de Enfermería, 29(3), 137-141. Retrieved from https:// scielo.isciii.es/scielo.php?pid=S1132$12962020000200008 \& \mathrm{script}=\mathrm{sci}$ arttext\&tlng=en

El Comercio. (2020). Solo el 40,1\\% de los hogares peruanos tiene acceso a Internet: ¿Qué hacer para elevar la conectividad? El Comercio Perú. Retrievedfromhttps://elcomercio.pe/economia/ peru/solo-el-401-de-los-hogares-peruanostiene-acceso-a-internet-que-hacer-para-elevarla-conectividad-congreso-velocidad-minimade-conexion-inei-ncze-noticia/

France 24. (2021). Con la mayor mortalidad, Perú supera los 200.000 fallecidos por Covid-19. Retrieved December 9, 2021, from https://www. france24.com/es/minuto-a-minuto/20211023- 
perú-muy-golpeado-por-la-pandemia-superalos-200-000-muertos-por-covid-19

Furman, M. (2020). Tres lecciones que nos deja la pandemia para transformar la educación. La Educación Del Mañana ¿inercia o Transformación?, 241-253. Retrieved from https://ri.conicet.gov.ar/ bitstream/handle/11336/139799/CONICET_ Digital_Nro.5bdee016-ed33-403e-9417bf016740f24e_B.pdf?sequence $=5$

García Aretio, L. (2020). COVID-19 y educación a distancia digital: preconfinamiento, confinamiento y posconfinamiento. RIED. Revista Iberoamericana de Educación a Distancia, 24(1), 09. https://doi.org/10.5944/ ried.24.1.28080

Gestión. (2020). Estas son las redes sociales más usadas desde los teléfonos móviles en Perú. Retrieved December 8, 2021, from https:// gestion.pe/tendencias/estas-son-las-redessociales-preferidas-por-usuarios-moviles-enel-peru-noticia/

Giraldo Ospina, G. A., Gómez Gómez, M. M., y Giraldo Ospina, C. F. (2021). COVID-19 y uso de redes sociales virtuales en educación médica. Educación Médica, 22(5), 273-277. https://doi. org/10.1016/j.edumed.2021.05.007

Gómez-Arteta, E.-M. (2021). Educación virtual en tiempos de pandemia: Incremento de la desigualdad social en Perú [Virtual education in times of pandemic: Increase in social inequality in Peru]. Biblioteca Electrónica Científica En Línea, I, 1-13. Retrieved from https://n9.cl/ e7czk

Grillo Rojas, P. F., y Romero Onofre, R. (2020). Estimate of the excess of the total deaths reported in 2020 versus the reported deaths from COVID-19 (SARS-CoV2) in Peru during the months of March, April and May 2020. Revista de La Facultad de Medicina Humana, 20(4), 646-650. https://doi.org/10.25176/rfmh. v20i4.3220

INEGI. (2019). En hogares. Retrieved from https:// www.inegi.org.mx/temas/ticshogares/
Leal Ortiz, N. (2021). Educación a Distancia desde la presencialidad en tiempos de pandemia. Educ@ción En Contexto, VII (14). Retrieved from https://educacionencontexto.net/journal/ index.php/una/article/download/158/295

Lovón Cueva, M. A., y Cisneros Terrones, S. A. (2020). Repercusiones de las clases virtuales en los estudiantes universitarios en el contexto de la cuarentena por COVID-19: El caso de la PUCP. Propósitos y Representaciones, 8(SPE3). https://doi.org/10.20511/pyr2020.v8nspe3.588

Morales Espíndola, M. G., Moreno Cortés, K. C., Romano Cadena, M. M. del S., y García Alarcon, M. del R. (2020). Gestión del conocimiento, a través de plataformas y herramientas digitales de aprendizaje ante la migración de clases presenciales a en línea. Revista Geon (Gestión, Organizaciones y Negocios), 7(2), 1-19. https:// doi.org/10.22579/23463910.217

Näslund-Hadley, E., Hernández-Agramonte, J. M., Namen, O., y Peña de Osorio, B. (2020). 3000 profesores comparten sus experiencias de aprendizaje remoto COVID-19 - Enfoque Educación. Retrieved December 9, 2021, from https://blogs.iadb.org/educacion/es/ docentescovid/

ONU. (2020). La pandemia es una oportunidad para repensar la educación y lograr un aprendizaje de calidad para todos. Retrieved December 8, 2021, from https://news.un.org/es/ story/2020/10/1481832

Pedraza Bucio, C. I. (2021). La brecha digital de género como vértice de las desigualdades de las mujeres en el contexto de la pandemia por Covid-19. LOGOS Revista de Filosofía, 136(136), 9-22. https://doi.org/10.26457/lrf. v136i136.2873

RPP. (2021). Educar en pandemia: ¿Cuál es la situación de los docentes en la nueva realidad educativa? | educación a distancia | aprendo en casa. Retrieved December 9, 2021, from https://rpp.pe/campanas/valor-compartido/ educar-en-pandemia-cual-es-la-situacion-delos-docentes-en-la-nueva-realidad-educativa- 
educacion-a-distancia-aprendo-en-casanoticia-1339116

Santamaría Ortega, C. (2020). La Inteligencia Emocional y la resiliencia docente en Convivencia Escolar. Retrieved from https://zaguan.unizar. es/record/10321/files/TAZ-TFM-2013-144.pdf

Torres, C. I. (2017). Ecosistemas digitales y su manifestación en el aprendizaje: Análisis de la literatura Digital ecosystems and their manifestation in learning: Analysis of literature. Retrieved from https://revistas.um.es/red/ article/download/315361/222291

UNESCO, C. (2020). Educación en tiempos de pandemia (covid-19). Revista Universidad de La Salle, 1(85), 51-59. https://doi.org/10.19052/ ruls.vol1.iss 85.4
Valverde Rodríguez, W. (2021). Trujillo en tiempos de pandemia COVID 19: relatos etnográficos sobre la ciudad, el trabajo y la vida. Revista Panameña de Ciencias Sociales, (5), 97-110. Retrieved from https://revistas.up.ac.pa/ index.php/rev_pma_ciencias_sociales/article/ download/2194/2033

Zapata, F., y Vidal, R. (2016). La investicación-acción participativa Guía conceptual y metodológica del Instituto de Montaña. Mountain. Pe, 58. Retrieved from https://pdf.usaid.gov/pdf_docs/ PA00N1QH.pdf 